\title{
IMPROVED FUSION METHOD FOR INFRARED AND VISIBLE IMAGE
}

\author{
Nitin Salvi, Darshali More, Krutika Thakur \\ B.E., Computer Engineering, \\ St. John College of Engineering and \\ Management, \\ Palghar, Maharashtra, India
}

\author{
Mrs. Vipra Dave \\ Assistant professor, \\ Department of Computer Engineering, \\ St. John College of Engineering and \\ Management, \\ Palghar, Maharashtra, India
}

\begin{abstract}
When images are being use for security purpose, such image should be much productive. To achieve this there are several techniques to improve corresponding image like, image filtering, image enhancement, image fusion, etc. Hence we are using the concept of image fusion with some advanced methods like, IHS transform and multi-wavelet transform. Hence the first unclear image is taking advantage of the second image which contains clear view of that place. Images captured in night-time, in dark places or in different illumination are made much clearer than the original by fusing the night-time image with the day-time image captured at same fixed position. But sometimes this fusion is not much enough to give desired output from an image, hence this fused image of day-time and night-time image is again fused with an infrared image. Infrared images has an advantage over normal visible images that, infrared image shows the temperature difference of the objects present in an image which in turn is an advantage at night-time. Often image fusion results in distortion of the image quality, to avoid this we are applying multi-wavelet transform on the final fused image. The multi-wavelet transform will decompose an image, which gives us freedom work on each partition of an image. The image after performing multi-wavelet transform we get a decomposed image which will needs to be some more sharper so we that every edge can be recognized immediately, hence we are applying an IHS transform on each decomposition(i.e. each pixel) of an image which will result in enhanced image than the original. IHS transform enhances an intensity of each pixel of an image.
\end{abstract}

Keywords: Image fusion, Day-time image, Night-time image, Infrared image, IHS transform, Multi-wavelet transform.

\section{INTRODUCTION}

An image has huge applications in various fields such as social media, medical applications, military applications, computer vision, security surveillance at railway station, offices, school and college, etc. Basically there are four types of image fusions, multi-modal image fusion, multi-temporal image fusion, multi-view image fusion and multi-focus image fusion. We are applying multimodal and multi-temporal image fusion, where multitemporal image fusion technique merges two images of same place, same sensor but from different time and multimodal image fusion technique merges two images of same place, same time but captured from different sensors. When images are used for security or investigation purpose, such an image should be much clear not only when captured in day-time but also when captured in night-time. Initially it is enough to fuse a night-time image with day-time image to enhance the visual quality of such image. But sometimes when people expect more from such image, then fusion with a day-time image is not enough, hence we have extended this idea by inclusion of infrared image. We get a new image by the fusion of day and night image, this fused image is again will be fused with an infrared image. Infrared image does not get affected by the darkness or lowlight, because they only work on temperature of a human body or any object present in an image. We are improving this image fusion by inclusion of two transformations during such image fusion process, i.e. IHS transform and multiwavelet transform.

\section{IMAGE FUSION METHODS}

\section{(i) SIMPLE MAXIMUM METHOD}

In this method et al.(2016) maximum value of intensity of each pixel from both of input images is considered for fusion. It can mathematically expressed as,

$F(x, y)=\operatorname{Max}[I 1(x, y), I 2(x, y)]$.

\section{(ii) SIMPLE AVERAGE METHOD}

In this method et al.(2016), for each pixel average is calculated by comparing with nearby pixels intensity 


\section{International Journal of Engineering Applied Sciences and Technology, 2019 \\ Vol. 3, Issue 10, ISSN No. 2455-2143, Pages 20-22 \\ Published Online February 2019 in IJEAST (http://www.ijeast.com)}

values and then this average of intensity value of each pixel from both of input image is taken for fusion.

$\mathrm{F}(\mathrm{x}, \mathrm{y})=\operatorname{Avg}[\mathrm{I} 1(\mathrm{x}, \mathrm{y}), \quad \mathrm{I} 2(\mathrm{x}, \mathrm{y})]$

\section{IHS TRANSFORM}

In this method an image from RGB model is initially converted to IHS model, where 'I' stands for intensity, ' $\mathrm{H}$ ' stands for Hue and ' $\mathrm{S}$ ' stands for Saturation. Intensity represents spatial information or grey level, Hue represents the colour itself, and saturation represents the purity of colour.

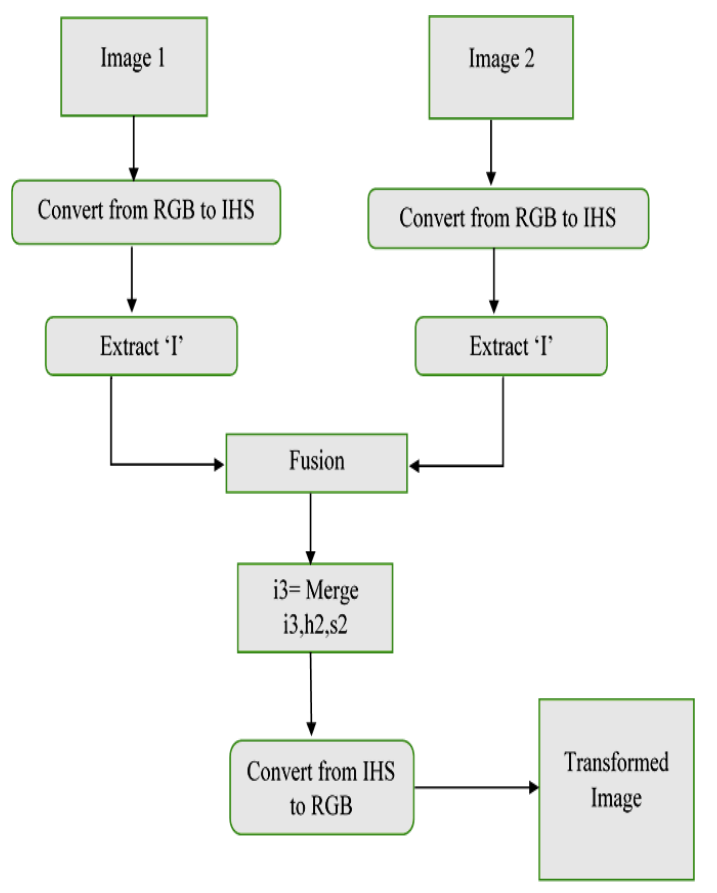

Figure 1. IHS Transform

As shown in figure 1, in IHS transform input images are split into three components i.e. I, $\mathrm{H}, \mathrm{S}$, which is intensity, hue and saturation respectively. Only ' $\mathrm{I}$ ' component from both the images are extracted for transformations. Then 'I' component of first image is added with 'I' component of second image, and forms a new image. But this image is still in IHS form, hence inverse IHS is performed to convert an image from IHS to RGB. After all this process, we get a new transformed high quality image.

\section{MULTI-WAVELET TRANSFORM}

Multi-wavelet transform et al.(2010) divides both the images in four co-ordinates named as, HHa, HLa, LHa, LLa for first image and HHb, HLb, LHb, LLb for second image. Then these co-ordinates of both the images are added together and we get four new co-ordinates named as, HHn, HLn, LHn, LLn, then the inverse multi-wavelet transform is performed on these new co-ordinates.
Figure 2 depicts the discrete wavelet transform, an image is passed through low pass filter and high pass filter and returns four coefficients (i.e. LL, LH, HL and HH) according to their colour bandwidth. After passing an image through low pass filter and high pass filter, discrete wavelet transform decomposes an image according to its four coefficients as given below:

i. Horizontal and vertical low frequency sub-band (LL)

ii. Horizontal low frequency and vertical high frequency sub-band (LH)

iii. Horizontal high frequency and vertical low frequency sub-band (HL)

iv. Horizontal high frequency and vertical high frequency sub-band (HH)

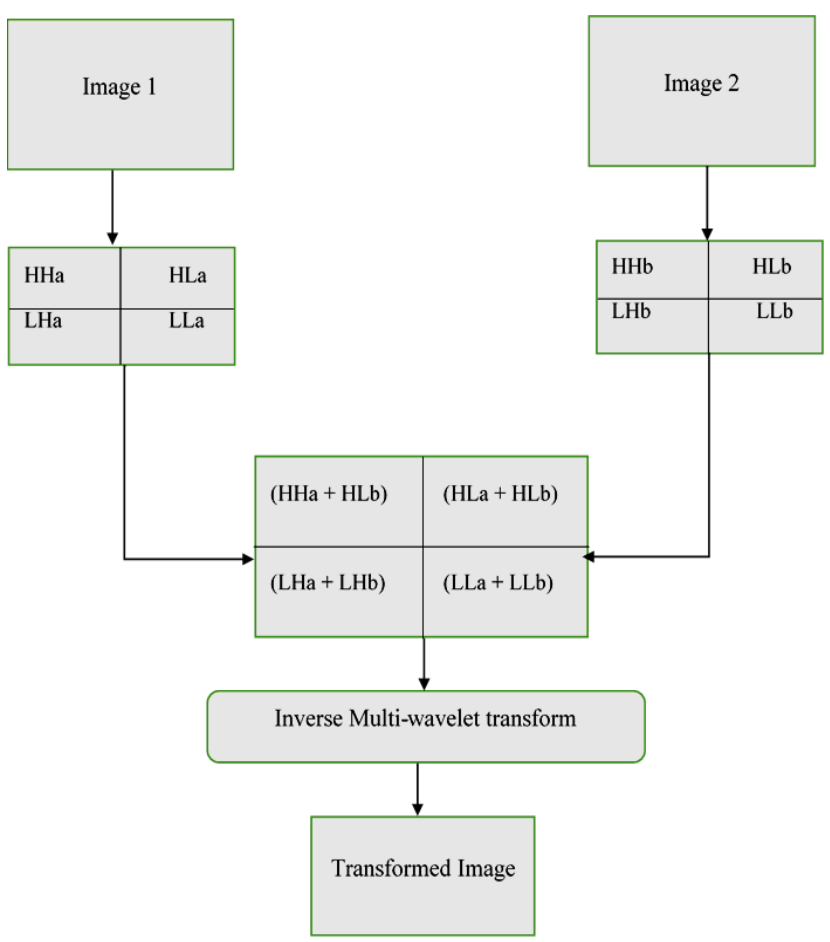

Figure 2. Multi-Wavelet Transform

The same process is again performed on each of this sub-band or sub-division as shown in figure 2. Multiwavelet transform, the same process is performed iteratively until there no possible division available. Then each ' $\mathrm{LH}$ ' and ' $\mathrm{HL}$ ' is replaced with its transformation, and then inverse multi-wavelet transform is performed to get a composite or restored image.

\section{IMAGE FUSION USING IHS AND MULTI- WAVELET TRANSFORM}

Figure 3, shows the approach to get the comparatively better quality of night time image captured in dark places. To achieve this basically we need to enhance an image, so that it will show the difference in comparison 


\section{International Journal of Engineering Applied Sciences and Technology, 2019 \\ Vol. 3, Issue 10, ISSN No. 2455-2143, Pages 20-22 \\ Published Online February 2019 in IJEAST (http://www.ijeast.com)}

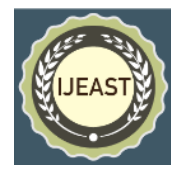

the original image. Hence we are using an IHS transform which in turn improves the image pixels by taking the advantage of another image. Figure 3 shows the combination of IHS and multi-wavelet transform used in this approach.

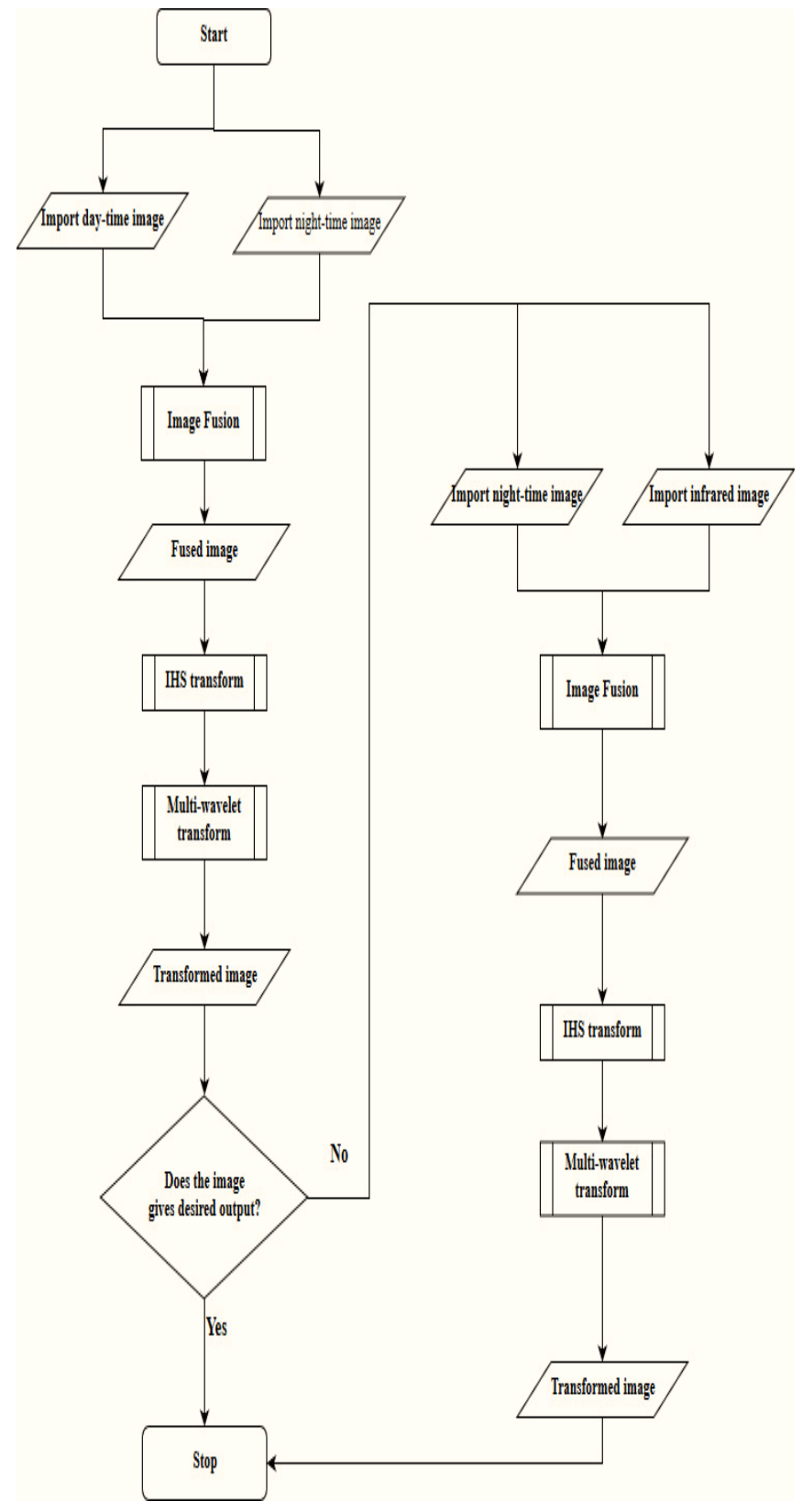

Figure 3. Implemented approach

\section{CONCLUSION}

Better transformation for the night-time image is to perform an image fusion on with the day-time image and further fused with an infrared image if needed, which is the key feature of the project. To overcome the drawbacks of image fusion, we are implementing an IHS transform and multi-wavelet transform. Multi-wavelet transform is most useful to prevent an image from distortion.

\section{REFERENCES}

[1] Tang Gouliang; Cai Zhongmin; Pu Jiexin; Huang Xin-Han, (C) 2010 IEEE,Color Daytime And Nighttime Image Fusion Algorithm Based On Ihs And Multi-Wavelet Transform, 978-1-4244-5141-8/10/\$26.00.

[2] Wu1 Ruiqing, 2, Yu1 Dayan, Liu1 Jian, Wu1 Hao, Chen1 Wei, Gu1 Qingshui, (C2017 IEEE, An Improved Fusion Method For Infrared And Low-Light Level Visible Image, 978-1-5386-1010-7/17/\$31.00.

[3] Iq. Pham And Polasek M., University Of Defence, Kounicova 65, 66210 Brno, The Czech Republic, (C2014 IEEE ,Algorithm For Military Object Detection Using Image Data, 978-1-4799-5001-0/14/\$31.00.

[4] Devi N.Vishnu, A.Sivasankar M.E. And S. Iswariya, July-2013international Journal Of Emerging Trends In Electrical And Electronics (Ijetee - Issn: 23209569), Multi-Temporal Image Fusion Using Subtraction Method, Vol. 5, Issue. 2.

[5] Mistry1 Mamta M, Vala2 Brijesh, 1m.E. Computer Engineering, Parul Institute Of Engineering And Technology, Gujarat, India, Assistant Professor, Parul Institute Of Engineering And Technology, Gujarat, India, Mar-2016, Survey On Different Image Fusion Techniques, Volume: 03 Issue: 03 .

\section{ACKNOWLEDGEMENT}

The success and final outcome of this project required a lot of guidance and support and also for the constant support from start to end which made us complete the project duly. We respect and thank Mrs. Vipra Dave, Assistant professor, Department of Computer Engineering, St. John College of Engineering and Management. 